\title{
Comportamiento al creep de un polímero utilizado para impresión 3D
}

\author{
Creep behaviour of a polymer \\ used for 3D printing
}

\author{
René Molina ${ }^{1}$, Gerardo Pender ${ }^{1}$, \\ Lilián Moro ${ }^{1}$, Marcelo Tulio Piovan ${ }^{2}$
}

\footnotetext{
${ }^{1}$ Grupo de Estudios de Materiales (GEMAT), Facultad Regional Bahía Blanca, Universidad Tecnológica Nacional. 11 de Abril 465, Bahía Blanca, Buenos Aires, Argentina. e-mail: rjmolina@ frbb.utn.edu.ar

2 Grupo de Análisis de Sistemas Mecánicos (GASM), Facultad Regional Bahía Blanca Universidad Tecnológica Nacional, 11 de Abril 465, Bahía Blanca, Buenos Aires, Argentina.

e-mail: gpender@frbb.utn.edu.ar, lmoro@frbb.utn.edu.ar, mpiovan@frbb.utn.edu.ar
}

\section{RESUMEN}

En los últimos años se está utilizando, ampliamente, la construcción aditiva o impresión 3D, para la fabricación de bienes tecnológicos. Este procedimiento de construcción tiene y tendrá un gran impacto industrial. Existen muchos procedimientos de impresión 3D y entre ellos el más conocido se denomina Modelado por Deposición de Filamento (MDF).

El objetivo del presente trabajo se orienta a la caracterización experimental de propiedades mecánicas de piezas construidas por impresión 3D con polímeros plásticos. Si bien ya se han efectuado varios estudios sobre la caracterización de propiedades mecánicas de piezas impresas por MDF, se debe mencionar que es poco lo evaluado relativo al fenómeno de creep o de termofluencia. En este trabajo se estudian las propiedades a la termofluencia del poli(ácido láctico) (PLA). Este polímero es termoplástico y biodegradable y se utiliza tanto en estado amorfo como cristalino, por lo que presenta numerosas aplicaciones tecnológicas.

Los ensayos de termofluencia por tracción, se efectúan a una temperatura de $303 \mathrm{~K}$, y a niveles de tensión correspondientes entre el 25\% y 50\% del límite de fluencia. Para ello, se emplean probetas estandarizadas construidas con diversos trazados de deposición del filamento plástico.

Palabras clave: MDF, PLA, Termofluencia, Resistencia mecánica

\section{ABSTRACT}

In recent years, additive construction or $3 \mathrm{D}$ printing technology is being widely used for manufacturing goods. This construction procedure has and will have a great industrial impact. There are several 3D printing processes, and among of them the most known is called Fused Deposition Modeling (FDM).

The objective of the present paper is oriented to the experimental characterization of the mechanical properties of pieces constructed by means of 3D printing using thermoplastic polymers. Although there are many studies about the characterization of mechanical properties in 3D printed parts, it should be noted that there is quite a little effort in evaluating the creep effect in 3D printed specimens. In this work the creep properties of the polylactic acid (PLA) are studied. This material is a thermoplastic and biodegradable biopolymer used in both amorphous and crystalline states, which has many technological applications.

The tensile creep tests are performed at a temperature of $303 \mathrm{~K}$ and at corresponding levels of tension between $25 \%$ and $50 \%$ of the yield strength of PLA. Standard specimens constructed with different layouts of plastic filament deposition are employed.

Keywords: FDM, PLA, Creep, Mechanical Strength.

\section{INTRODUCCIÓN}

Durante la década de 1980 a 1990 se desarrolló la técnica de impresión 3D por deposición de filamento, no 
obstante en los últimos años, se ha incrementado su utilización debido a la apertura comercial que se produce por el cese de las patentes industriales. Dentro de esta técnica se conocen distintos métodos para realizarla, los cuales presentan tanto ventajas como limitaciones para su uso [1].

El Modelado por Deposición de Filamento (MDF) es uno de los métodos más utilizados tanto desde el punto de vista profesional como en el semi-profesional, debido a la proliferación de impresoras de más accesible costo. En este proceso se empuja el material, a través de una boquilla de extrusión que, siguiendo un procedimiento de fabricación por control numérico (en código G), irá depositando capa a capa el material que conformará la pieza. Para que la impresión tenga lugar de una manera efectiva, el material extruido debe poseer una consistencia semi-sólida en el momento de abandonar la boquilla de extrusión. Del mismo modo, también se requiere que el material solidifique de una manera más o menos acelerada para que pueda soportar el peso de las capas posteriores y solidificar en conjunto con las capas anteriores. Así se conseguirá que la estructura tenga mucha más consistencia. La disposición de los filamentos en cada una de las capas permite que se asemeje, en parte, al modelo de refuerzo fibroso en láminas de material compuesto, tanto si poseen orientación en ángulo o bien a una configuración en panal de abeja u otros refuerzos estructurales internos.

En los primeros tiempos del uso de esta técnica, el aspecto constitutivo no se consideraba de importancia ya que los objetos construidos, solo se utilizaban como prototipos de evaluación visual y en forma previa a su construcción definitiva por otros procedimientos más tradicionales (corte o moldeo, entre otros). Sin embargo, en la actualidad y con el afán de optimización de costos de diseño y producción, se considera la impresión 3D como una herramienta versátil para la construcción de piezas complejas, útiles y funcionales, como componentes o partes de productos intermedios o como bienes comerciales definitivos [2].

Si bien se han encontrado numerosos estudios realizados sobre distintos polímeros plásticos con el fin de caracterizar las propiedades mecánicas de piezas obtenidas a partir del método de impresión 3D por MDF, no se han encontrado trabajos donde se evalúe el comportamiento a la termofluencia (creep) [3] de estos materiales una vez construidos por impresión 3D. Se debe destacar que la mayoría de los trabajos de investigación de los últimos dos a cinco años está vinculada a la determinación de propiedades elásticas ante solicitaciones estáticas convencionales (tracción, flexión, etc.) adaptando estándares internacionales propios de ensayos de plásticos moldeados por inyección.

En este trabajo se estudian las propiedades al creep del poli(ácido láctico) (PLA) que por ser un polímero termoplástico y biodegradable, su utilización presenta amplia ventajas respecto de otros polímeros que se encuentran en el mercado. Fundamentalmente resulta de interés, debido a la baja energía y bajo consumo de agua que se requiere durante su fabricación y la escasa emisión de $\mathrm{CO}_{2}$ que se produce. El PLA se obtiene a partir del ácido láctico y se utiliza tanto en estado amorfo como cristalino, siendo su estructura química la que se indica en la Figura 1 [4].<smiles>CCC(C)OC(=O)C(C)OC(=O)C(=O)O</smiles>

Figura 1: Estructura molecular del poli(ácido láctico) (PLA).

\section{MATERIALES Y MÉTODOS}

Se realizaron ensayos de termofluencia con un equipo de tracción donde se mantiene la tensión y temperatura constantes. El equipo consta de una barra principal pivotante, con una leva de radio constante en su brazo de menor longitud y un peso de trazabilidad controlada en su brazo mayor que permite el desplazamiento sobre el mismo para lograr la tensión elegida, en función de la distancia al centro de pivote. La deformación de la zona calibrada de la probeta, se mide por medio de dos transformadores diferenciales de variación lineal (LVDT) marca Solartron con una sensibilidad de $0.01 \mathrm{~mm}$, que produce una salida de corriente continua con una amplitud proporcional al desplazamiento de un núcleo movible. La señal de salida de los LVDT es adquirida por un sistema analógico- digital. En la Figura 2 se aprecia la máquina completa y el detalle del dispositivo de censado con los LVDT montados y ubicados dentro de la cámara de aislación térmica. 


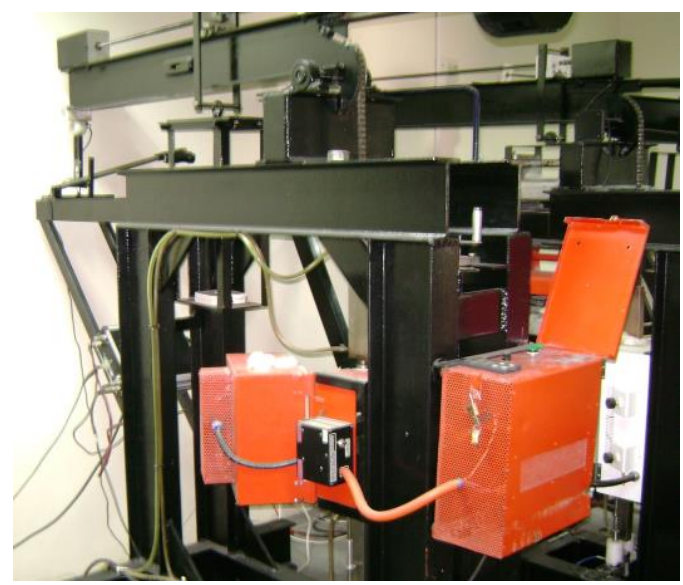

(a)

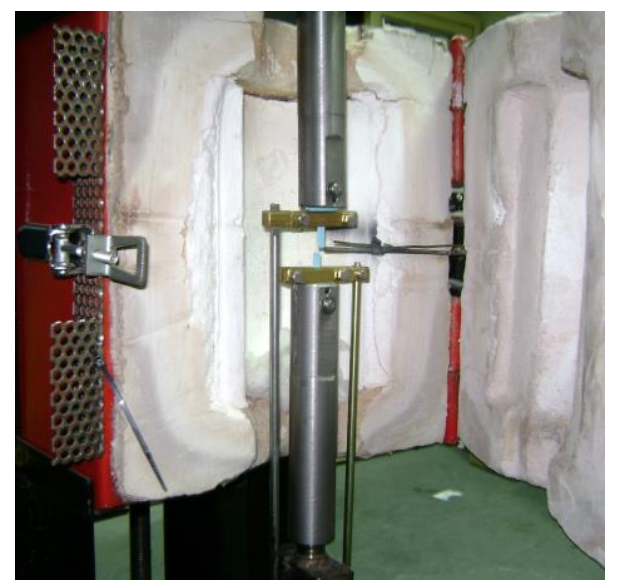

(b)

Figura 2: Máquina de ensayo creep. a) Vista general; b) Detalle de los LVDT

Las probetas se construyeron por el método MDF, utilizando una impresora 3D tipo open-source de marca registrada Replikat $\odot$, que usa el programa Kisslicer $\bigodot$. La impresora posee una boquilla de extrusión con diámetro de $0.4 \mathrm{~mm}$ y permite una resolución de capas de entre $0.1 \mathrm{~mm}$ a $0.3 \mathrm{~mm}$. En la Figura 3 se aprecia la geometría de las probetas empleadas con las dimensiones más importantes y necesarias para este estudio. El protocolo de impresión 3D fue fijado en una temperatura de $195^{\circ} \mathrm{C}$ para el extrusor y de $60^{\circ} \mathrm{C}$ para la plataforma de deposición, la velocidad de impresión fue de $40 \mathrm{~mm} / \mathrm{s}$, mientras que la resolución de las capas fue seleccionada en $0.25 \mathrm{~mm}$. Se construyeron dos tipos de probetas con distinta textura. En la primera textura las capas son conformadas con los filamentos en dirección longitudinal en la zona calibrada, mientras que en la segunda los filamentos se orientan en una dirección que forma un ángulo de $45^{\circ}$ respecto de los filamentos del primer caso. En ambos casos el llenado (o Infill) del volumen es completo.

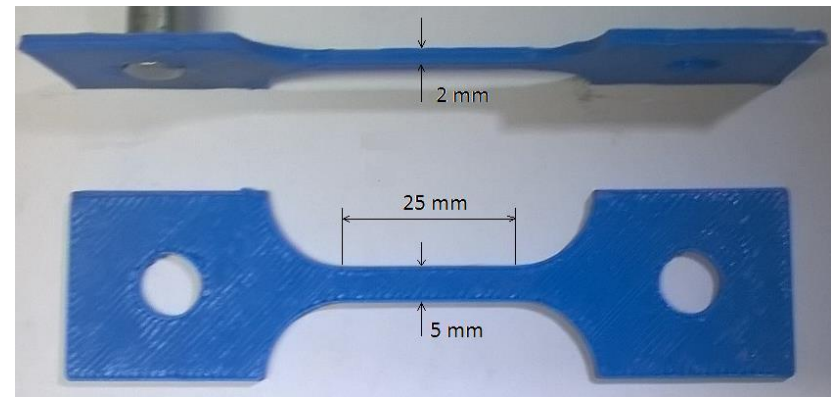

Figura 3: Geometría de las probetas.

Se realizaron ensayos de creep hasta la rotura, fijando una temperatura de $303 \mathrm{~K}$ y tensiones efectivas de 10, 20 y $30 \mathrm{MPa}$, manteniendo ambas variables constantes durante cada ensayo. Se determina la velocidad de deformación equivalente, que se mantiene constante en la etapa del creep secundario, la cual se vincula con la temperatura y la tensión aplicadas, a partir de la ecuación empírica o ley de potencias que se expresa como:

$$
\dot{\varepsilon}=\mathrm{A} \sigma^{\mathrm{n}} \mathrm{e}^{\Delta \mathrm{Q} / \mathrm{RT}}
$$

donde: $\dot{\varepsilon}$ es la velocidad de deformación equivalente, $A$ una constante función de la estructura, $\mathrm{n}$ el exponente de tensión, $Q$ la energía de activación del creep, $R$ la constante universal de los gases y $T$ la temperatura absoluta [4].

\section{RESULTADOS Y DISCUSIÓN}

Para evaluar el comportamiento del material se trazan las curvas de la deformación equivalente en función del tiempo para los ensayos realizados (Figura 4). En la Tabla 1 se indican los valores de la velocidad de deformación de la etapa secundaria, el tiempo de rotura y la deformación porcentual al finalizar cada ensayo, en este caso para probetas con textura de construcción orientada en la dirección de tracción. 
Tabla 1: Resultados obtenidos de los ensayos de creep a $303 \mathrm{~K}$

\begin{tabular}{l|l|l|l}
\hline $\begin{array}{l}\text { TENSIÓN } \\
\text { (MPa) }\end{array}$ & $\begin{array}{l}\text { VELOCIDAD DE } \\
\text { DEFORMACIÓN }\end{array}$ & $\begin{array}{l}\text { TIEMPO DE } \\
\text { ROTURA (h) }\end{array}$ & $\begin{array}{l}\text { DEFORMACIÓN DE } \\
\text { ROTURA (\%) }\end{array}$ \\
\hline 10 & $1,7417.10^{-7}$ & 723,16 & 11,7 \\
\hline 20 & $4,1666 \cdot 10^{-7}$ & 52,84 & 12,88 \\
\hline 30 & $6,7307.10^{-6}$ & 1,11 & 4,68 \\
\hline
\end{tabular}

Se observa que trabajando a tensiones de 10 y $20 \mathrm{MPa}$ los resultados son muy similares, pero cuando se somete al material a una tensión de $30 \mathrm{MPa}$ hay una gran desviación del comportamiento, ya que aumenta la velocidad de deformación y disminuye notablemente el tiempo de ensayo de forma que en corto tiempo se encuentra en la etapa de creep terciario. Se supone que, a esta tensión de trabajo, en el material se produce un reordenamiento de las cadenas del polímero y ruptura de los dobles enlaces.

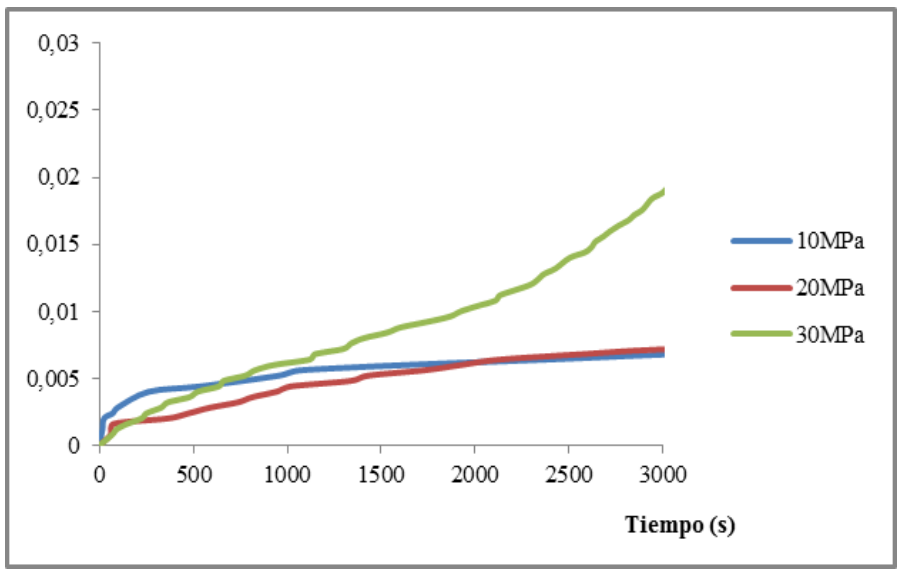

Figura 4: Gráfica de la deformación especifica por creep en función del tiempo.

Esta diferencia de comportamiento también se observa al estudiar las características del tipo de fractura en cada uno de los casos. En la figura 5 se muestra una comparación entre cada una de las probetas, donde la inferior corresponde al ensayo de $10 \mathrm{MPa}$ y la superior al de $30 \mathrm{MPa}$. Se puede observar que para las dos primeras tensiones la rotura se produce según un plano normal al eje longitudinal de la probeta y dentro del tercio medio, pero que al incrementarse la tensión a $30 \mathrm{MPa}$ la fractura se presenta formando un ángulo aproximado a $\operatorname{los} 45^{\circ}$.

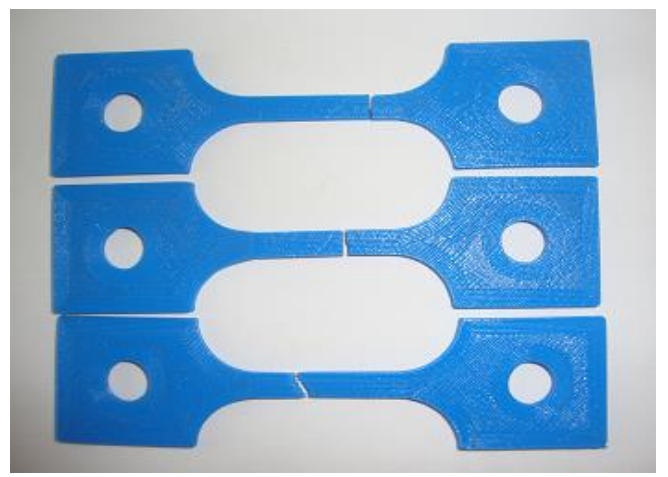

Figura 5: Forma de fractura de las probetas a 10, 20 y $30 \mathrm{MPa}$

Se realizó también una observación macrográfica de las secciones transversales de cada probeta comparando, según se muestra en la Figura 6, el material sin ensayar con las muestras que previamente fueron ensayadas a tensiones de 20 y $30 \mathrm{MPa}$. En Figura 6 (a) se observa la integridad estructural de la sección transversal de la probeta sin ensayar y que sirve de referencia para evaluar la degradación estructural para cada uno de los otros ensayos. Así en la Figura 6 (b) se aprecia que para la tensión de $20 \mathrm{MPa}$ se verifica un 
plano de fractura con presencia de cavidades generadas entre los filamentos de material y en la Figura 6 (c), que muestra la superficie del material ensayado a $30 \mathrm{MPa}$, y donde se observa la notable deformación sufrida por los hilos de material y la coalescencia de cavidades.

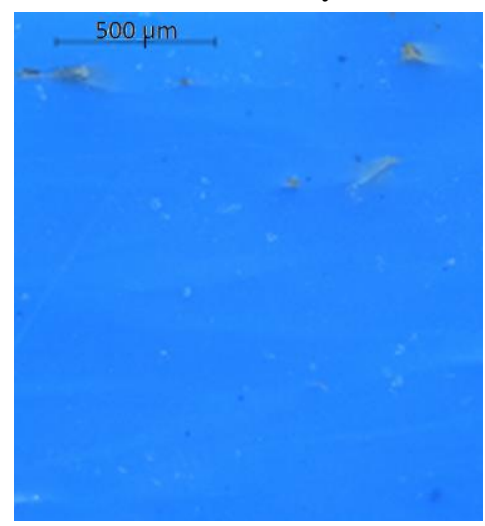

(a)

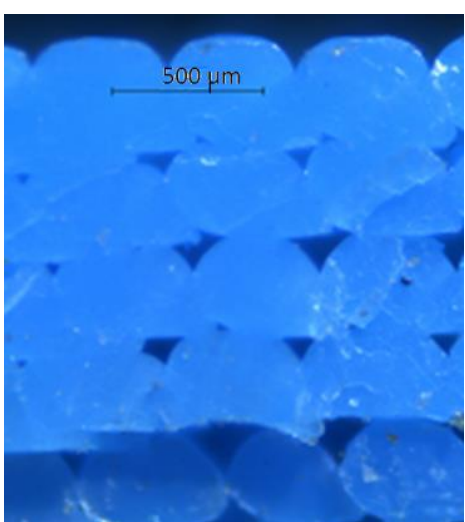

(b)

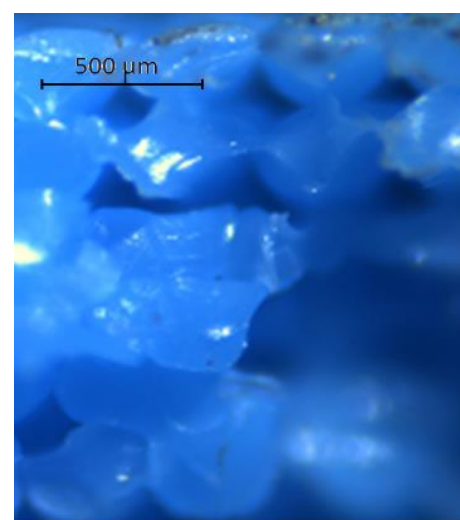

(c)

Figura 6: Macrografías que muestran la superficie de fractura del material. (a) Sin ensayar; (b) Ensayado a $20 \mathrm{MPa}$; (c) Ensayado a $30 \mathrm{MPa}$. (50X)

Por último, se comparó el comportamiento al creep del PLA cuando se depositó en forma longitudinal, es decir con las fibras en una dirección paralela al eje de tracción con el caso donde los filamentos se presentan orientados a $45^{\circ}$ respecto del eje de tracción. En ambas situaciones se realizaron ensayos de creep a una temperatura de $303 \mathrm{~K}$ y una tensión de $20 \mathrm{MPa}$, en la Figura 7 se observan las curvas de la deformación específica en función del tiempo para ambas texturas. Se observa que las probetas con disposición a $45^{\circ}$ respecto del eje de tracción tienen una deformación que llega a superar, dentro de la etapa secundaria de creep, en más de 5 veces la correspondiente de las probetas con disposición de filamentos co-lineal al eje de tracción. Este comportamiento tiene un cierto paralelismo con el homónimo problema de materiales compuestos laminados de resinas reforzadas por fibras y su uso en estructuras [6]; puesto que la disposición de fibras en ángulo con respecto a la dirección de forzamiento tiene una menor resistencia mecánica y por ende mayor tendencia a deformación que su contraparte co-lineal al forzamiento. Aun así se debe destacar que construcciones en capas hechas por impresión 3D no son en esencia semejantes a las de materiales compuestos laminados puesto que estas últimas poseen matriz resinosa y las primeras no.

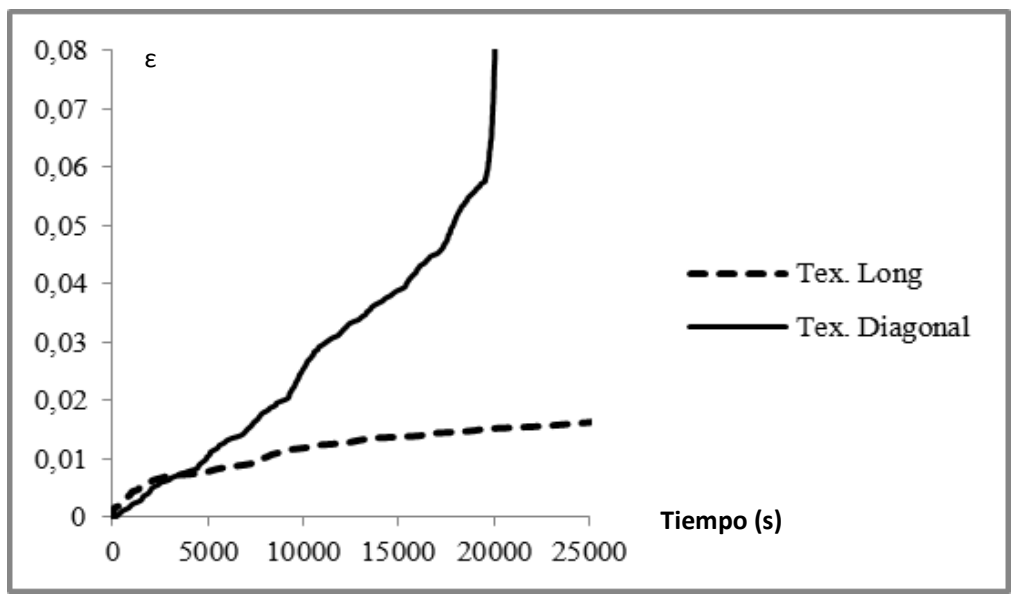

Figura 7: Gráfica de la deformación específica por creep en función del tiempo, comparando las dos configuraciones constructivas.

En la Tabla 2 se indican los valores de la velocidad de deformación de la etapa secundaria, el tiempo de rotura y la deformación porcentual para cada configuración constructiva al finalizar el ensayo. Se observa una mayor resistencia en la configuración longitudinal. 
Tabla 2: Resultados obtenidos de los ensayos de creep a 303K y 20MPa para las dos configuraciones.

\begin{tabular}{l|l|l|l}
\hline TEXTURA & $\begin{array}{l}\text { VELOCIDAD DE } \\
\text { DEFORMACIÓN }\end{array}$ & $\begin{array}{l}\text { TIEMPO DE } \\
\text { ROTURA (h) }\end{array}$ & $\begin{array}{l}\text { DEFORMACIÓN DE } \\
\text { ROTURA (\%) }\end{array}$ \\
\hline Longitudinal & $4,1666.10^{-7}$ & 52,84 & 12,88 \\
\hline Diagonal & $2,8082.10^{-6}$ & 5,57 & 10,64 \\
\hline
\end{tabular}

\section{CONCLUSIONES}

A partir de los estudios realizados se puede concluir que:

- Las probetas de PLA que se someten a termofluencia a una temperatura de 303K evidencian una importante disminución de su resistencia mecánica cuando la tensión aplicada es mayor a $20 \mathrm{MPa}$.

- Para esta situación la fractura se presenta formando un ángulo de aproximadamente $45^{\circ}$ respecto del eje de tracción.

- A partir del estudio macrográfico se evidenció separación de las fibras y coalescencia de cavidades que se suponen relacionadas al reordenamiento de las cadenas de polímero y a la ruptura de los dobles enlaces.

- Se ha determinado que las probetas con orientación de las fibras en forma paralela al eje de tracción presentan una mayor resistencia al creep respecto de la distribución que se presenta en forma oblicua al eje.

A partir de este conjunto de conclusiones y con el material experimental disponible, es posible concebir un modelo matemático que responda al comportamiento viscoelástico (al menos en las etapas primaria y secundaria), para facilitar el diseño y análisis de piezas construidas por impresión 3D.

\section{AGRADECIMIENTOS}

Los autores desean agradecer a la Secretaría de Ciencia y Tecnología de la Universidad Tecnológica Nacional, la cual ha financiado el presente trabajo de investigación merced al proyecto TUN4285.

\section{BIBLIOGRAFÍA}

[1] CANESA E., FONDA C., GENARO M., Low cost 3D-printing, Ed. Int. Center Theor. Physics, 2013.

[2] FRANCE, A, K., Make 3D printing, Ed. Maker Media, Inc., 2014.

[3] TYMRAK, B.M., KREIGER, M., PEARCE, J.M., "Mechanical properties of components fabricated with open-source 3-D printers under realistic environmental conditions", Materials and Design, v. 58, pp. 242-246, 2014.

[4] AURAS R., LIM L.T., SELKE S.E.M., TSUJI H., Poly(Lactic Acid): Synthesis, Structures, Properties, Processing, and Applications, Wiley \& Sons, Inc. 2010.

[5] KASSNER, M.E., PEREZ-PRADO, T., Fundamentals of Creep in Metals and Alloys, 2nd Ed, Elsevier, 2004.

[6] PIOVAN, M.T., CORTINEZ, V.H., "Linear viscoelastic analysis of straight and curved thin-walled laminated composite beams", International Journal of Solids and Structures, v.45, pp.3466-3493, 2008. 